\title{
Priorización curricular en pandemia: oportunidad de un nuevo curriculum escolar en Chile
}

\author{
Curricular Prioritization in a Pandemic Context: Opportunity \\ for a New School Curriculum in Chile
}

Cristián Venegas Traverso*

\section{RESUMEN}

El trabajo describe las características del curriculum oficial vigente en Chile y del proceso de priorización curricular en el contexto de pandemia por covid-19 para los años 2020-2021. Además, argumenta la necesidad de construir un curriculum nacional menos denso en metas educativas, favoreciendo una mayor autonomía curricular de las comunidades educativas, entre otros.

El artículo se estructura en una contextualización del sistema escolar chileno en el escenario covid-19, una revisión teórica y normativa del curriculum oficial, la descripción de la propuesta de priorización curricular en educación básica elaborada por el Ministerio de Educación, y la presentación de las desventajas del actual curriculum escolar y los beneficios de una nueva arquitectura.

\section{ABSTRACT}

This study describes the characteristics of the official curriculum in place in Chile and the curricular prioritization process in the context of the Covid-19 pandemic for the 2020 and 2021 school years. It also argues for the need to construct a national curriculum that is less dense in educational goals and favors a greater curricular autonomy of educational communities, among others.

The article is structured such that it provides a contextualization of the Chilean school system during the Covid-19 pandemic, a
Palabras

clave: política

educacional, programa de enseñanza, plan de estudios, autonomía educativa, educación básica

Key words:

educational policy, teaching program, study plan, educational autonomy, elementary education. 
Priorización curricular en pandemia: oportunidad de un nuevo curriculum escolar | Venegas

theoretical and regulatory review of the official curriculum, a description of the curricular prioritization proposal in elementary education prepared by the Ministry of Education, and the presentation of the disadvantages of the current school curriculum and the benefits of a new architecture. 


\section{Antecedentes contextuales: pandemia y curriculum escolar chileno}

En el mundo, los procesos escolares se han visto fuertemente interrumpidos tras la declaración del covid-19 como pandemia por la Organización Mundial de la Salud (OMS), en marzo de 2020 (Mayo Clinic, s/f).

En Chile, el primer caso covid-19 fue identificado el 3 de marzo, un día antes del inicio del año escolar 2020. Casi dos semanas después, ante el rápido aumento de contagios en el país, el gobierno chileno decidió suspender las clases, primero por dos semanas y, luego, el 30 de marzo, por quince días más. Además, se determinó adelantar las vacaciones de invierno del 13 al 24 de abril (CNN Chile, 2020). Las muertes asociadas al virus, la implementación de cuarentenas y toques de queda en todo el país, más el cierre de fronteras y otras medidas, obligó a suspender indefinidamente las clases presenciales de educación inicial, básica y media desde el 27 de abril ${ }^{1}$.

En ese momento se contemplaba, desde el gobierno, la reapertura gradual de los establecimientos en un corto plazo, y cuanto más para el segundo semestre de 2020. Mientras tanto, con el fin de mantener el proceso educativo vigente, se promovió el trabajo escolar a través de medios virtuales (educación remota), estimándose necesario contar con un curriculum más liviano y flexible a las nuevas condiciones de enseñanza. El cambio de modalidad de presencialidad a virtualidad y la reducción curricular fueron acciones compartidas transversalmente por los países latinoamericanos y del Caribe, no obstante considerando que el impacto educativo sería alto (CEPAL-UNESCO, 2020).

En este marco, el Ministerio de Educación de Chile (MINEDUC) inició una revisión de los Objetivos de Aprendizaje (OA) del curriculum escolar, proyectando concentrar la enseñanza de contenidos esenciales en un periodo de tiempo más acotado que las 38 semanas que componen un año escolar. Su resultado fue una propuesta curricular de OA prioritarios (de $1^{\circ}$ y $2^{\circ}$ nivel) por curso y asignatura, que pudiera guiar a las escuelas sobre las expectativas mínimas de aprendizaje, para que fueran ajustados a sus posibilidades y realidad (MINEDUC, 2020b).

1 Además, se dispuso de una plataforma Aprendo en línea, con material y recursos para estudiantes de educación básica y media y acceso a la Biblioteca Nacional Escolar (MINEDUC, 2020a) 
La priorización curricular, cuya implementación abarcaría el periodo 2020-2021 para retomar en 2022 las Bases Curriculares (BC) vigentes, proponía a docentes y escuelas:

avanzar por un primer nivel de objetivos (de aprendizaje) reducidos que corresponde a los objetivos imprescindibles, aquellos considerados esenciales para avanzar a nuevos aprendizajes. Estos objetivos actuarán como un primer nivel mínimo que permitirá a las escuelas organizarse y tomar decisiones de acuerdo con las necesidades y reales posibilidades en el actual contexto. (MINEDUC, 2020b, p. 13).

Oficialmente, la producción de la priorización curricular aguardó tres criterios (MINEDUC, 2020b):

I. Equilibrio entre objetivos de los ejes de cada asignatura, a favor de la articulación curricular entre niveles y asignaturas.

II. OA priorizados coherentes a la progresión de objetivos durante el ciclo y que facilitan el aprendizaje.

III. Un grupo de OA priorizados imprescindibles para continuar el aprendizaje del año escolar siguiente (Nivel 1), y el otro, objetivos que resultan altamente "integradores y significativos", que amplían los objetivos de primer nivel (Nivel 2).

A su vez, la elaboración de la priorización aguardó cinco pasos (MINEDUC, 2020b):

Paso 1. Identificación en cada eje de OA imprescindibles, confirmando un equilibro de objetivos por eje: ¿Cuál es el aprendizaje fundamental que necesita desarrollar el estudiante para avanzar en los dominios de la asignatura? ¿Cuáles son los aprendizajes esenciales que debiese dominar el estudiante al terminar el año?

Paso 2. Construcción de la progresión de los objetivos prioritarios aplicando el criterio "imprescindibles".

Paso 4. Fundamentación de los objetivos que no fueron priorizados: ¿El objetivo puede desarrollarse a través de otro objetivo que haya sido priorizado? ¿El objetivo puede desarrollarlo el estudiante en contexto de aprendizajes informales?

Paso 5. Elaboración de orientaciones didácticas para la enseñanza de cada objetivo priorizado. 
Desde un plano crítico de los criterios de selección, se denota la ideación de un curriculum que, en parte, desconoce el tiempo-espacio de su desarrollo, al no referir a las necesidades socioeducativas que la pandemia ha generado, enmarcando la deliberación curricular en requisitos instrumentales o de orden técnico, paradigma recurrente en el quehacer curricular chileno (Cárdenas, Guerrero \& Johnson, 2021).

En la misma línea, el Informe COVID-19 CEPAL-UNESCO planteó la urgencia de responder con los currículos a la coyuntura pandémica, escogiendo aquello que permita al estudiantado:

lograr una mejor comprensión de la crisis y responder a ella de mejor forma, incorporando aspectos relativos al cuidado y la salud, el pensamiento crítico y reflexivo en torno a informaciones y noticias, la comprensión de dinámicas sociales y económicas, y el fortalecimiento de conductas de empatía, tolerancia y no discriminación, entre otros. [...] Igualmente, es necesario incorporar un enfoque de género, que permita visibilizar y erradicar situaciones de desigualdad o violencia de género que podrían estar viéndose profundizadas en el contexto de confinamiento. (CEPALUNESCO, 2020, p. 4).

Sin embargo, entre los países de los miembros de la OCDE se detectaron preferencias heterogéneas sobre lo priorizado curricularmente:

Algunos países y centros educativos han optado por dar preferencia al contenido curricular básico, que es esencial para la progresión de los alumnos y para que puedan pasar los exámenes, centrándose a menudo en la lectoescritura y la aritmética elemental; otros países consideran que la crisis ha demostrado la necesidad de fomentar una gama de habilidades cognitivas, sociales y emocionales más amplia, y se centran en el bienestar de los alumnos. (Reimers \& Schleicher, 2020, p. 7).

Volviendo al caso chileno, la priorización curricular presentó un plan de implementación sostenido en tres pilares (seguridad, flexibilidad y equidad), desarrollado en tres etapas (MINEDUC, 2020b):

ETAPA I. Diagnóstico integral. Aplicación de cuestionarios de diagnóstico socioemocional al universo de estudiantes, para identificar su estado y disposición frente a la pandemia, y la ejecución de evaluaciones en Lectura y Matemática entre $2^{\circ}$ básico y $\mathrm{II}^{\circ}$ medio. 
ETAPA II. Plan de reforzamientos de los aprendizajes y evaluación formativa. Orientaciones didácticas para nivelar el aprendizaje según los resultados del Diagnóstico Integral.

ETAPA III. Plan de trabajo con el curriculum transitorio. Aplicación del curriculum priorizado entre 2020 y 2021, y entrega a directivos, docentes y estudiantes, de recursos y orientaciones pedagógicas y de gestión, considerando inéditamente la exhibición de material educativo en televisión abierta y radios.

El 13 de mayo de 2020 el Consejo Nacional de Educación (CNED) aprobó formalmente la propuesta de Priorización Curricular de MINEDUC. En su justificación, CNED sostiene claramente que la priorización es solo una propuesta curricular para las escuelas y no reemplaza a las BC, las que encuentran vigentes (MINEDUC, 2020c).

Sobre el proceso de construcción curricular, a la fecha solo ha sido posible reconocer a la Unidad de Curriculum y Evaluación de MINEDUC como autora del curriculum priorizado, sin advertirse la colaboración de especialistas e instituciones, públicas y privadas, y comunidades escolares. Esto no es menor, en el supuesto de que su carácter reservado obstaculizaría la pertinencia y apropiación de la propuesta entre los actores del sistema escolar chileno, manteniendo la centralización de las decisiones curriculares (Cárdenas et al., 2021).

Inversamente, la entidad gremial Colegio de Profesoras y Profesores de Chile (CPC) levantó la nuclearización curricular como una salida alternativa a la priorización propuesta de MINEDUC. La nuclearización implica la construcción curricular desde núcleos temáticos, confluyendo OA de asignaturas diversas, en un sentido holístico, para la "comprensión global del mundo". Para el CPC (2020) la priorización supone simplemente el recorte o poda curricular, bajo criterios discutibles de inclusión/exclusión y dando continuidad a la lógica de cobertura y eficacia escolar haciendo simplemente ajustes por criterios formales de temporalidad. La propuesta del CPC no tuvo acogida oficial.

$\mathrm{Al}$ respecto, CEPAL-UNESCO (2020) considera que la nuclearización requiere de una alta valoración a la autonomía docente, además del desarrollo de altas competencias. Ante esto, bien cabe preguntarse si la negación de la autoridad chilena a la nuclearización y a un mayor 
protagonismo del magisterio en el proceso de selección curricular estaría revelando una baja estima de sus capacidades.

En tanto, la propuesta de nuclearización levantada por el CPC tuvo un importante hito previo a la pandemia, con las recomendaciones de la Mesa de Desarrollo Curricular (MDC) convocada por MINEDUC entre 2015 y 2016. Precisamente, la instancia propuso que los objetivos educativos se expresasen en aprendizajes nucleares, anuales y progresivos, para resguardar que el curriculum escolar sea cubierto en su extensión (MINEDUC, 2016), regulando o disminuyendo su densidad de expectativas formativas.

Sin embargo, es conveniente aclarar que nuclearización y priorización no son enfoques opuestos. Es más, durante 2020 no pocos países de la región combinaron ambas modalidades (CEPAL-UNESCO, 2020). Son dos formas de organización curricular que responden a distinta urgencia, pero que se reconocen como alternativas de arquitectura formativa, las que pueden ser exploradas en virtud de las deficiencias curriculares que el sistema ha ofrecido para las comunidades educativas, antes y después de la pandemia.

\section{El curriculum oficial y la práctica curricular}

En países como Chile, el curriculum escolar, oficial o nacional, responde a un tipo de diseño elaborado y distribuido por el Estado a los establecimientos del sistema escolar, cuya aplicación lectiva es obligatoria (Magendzo, 2008). Supuestamente, en términos generales, la ejecución forzosa del curriculum oficial permite dar estabilidad y sentido de largo plazo a las políticas públicas en educación (UNICEF, 2013), además de asegurar una justicia curricular, al trabajar sobre un "guión común" que evita la desigualdad, y no sobre lo que la capacidad e interés de cada docente permita (Dussel, 2020).

Asimismo, el curriculum oficial, mediante bases curriculares y planes y programas de estudio, expresa la formación mínima de los sujetos en el Estado, convirtiéndose en un eje vinculante entre el sistema escolar y la proyección futura de una sociedad (UNICEF, 2013; Castillo \& Salgado, 2018). Para esto, el curriculum oficial produce un "macro bosquejo" de lo que una sociedad ha definido como legado para sus generaciones posteriores, siendo por tanto una herramienta estraté- 
gica y política (Amadio, Opertti \& Tedesco, 2015) y "una expresión de poder para influir a las próximas generaciones" (Pinar, 2014, citado en Inostroza \& Lohaus, 2019, p. 153).

La producción del curriculum oficial tiene como finalidad natural la aplicación práctica en la escuela y el aula, sin embargo, este proceso no es ni ha sido armónico.

Existe una pugna entre la centralización curricular, expresada por las disposiciones curriculares del Estado hacia los establecimientos, y la descentralización, exigida por las comunidades escolares, en especial por el profesorado (Magendzo, 2008). En efecto, "la distancia entre lo que ha sido puesto en 'papel' y aquello que se hace 'vida' en las aulas es un punto crítico, tanto para el deseo de una implementación efectiva por parte de los responsables del sistema escolar, cuanto para el desarrollo de la autonomía profesional de los profesores" (Cárdenas et al., 2021, p. 40).

Respecto de otras pugnas educativas, este conflicto es relativamente nuevo, acaso superior a un siglo. La idea de un currículo nacional vino a instalarse recién a principios del siglo XX (Tadeu da Silva, 1999; Díaz Barriga \& García, 2014), y con este surgen los sistemas educativos centralizados, para formar mano de obra calificada y construir la identidad nacional, entre otros fines (Díaz Barriga \& García, 2014; Amadio et al., 2015). Previamente, la educación formal y el curriculum estaban en un terreno relativamente autónomo para las escuelas respecto del Estado, por lo que la tirantez entre centralización y autonomía escolar era mínima.

Para el curriculum oficial, no solo su aplicación práctica en el aula es su razón de ser. Su éxito se juega más en las escuelas, comunidades educativas y salas de clase, y en la gestión docente y directiva que en los espacios reservados del Estado en donde se define controlada y técnicamente lo que enseñará. Consecuentemente, la OCDE (2017, citado en Arratia, Vargas \& Latorre, 2018) expresa que "no importa cuán visionario sea un curriculum, solo será útil en la medida en que las escuelas tengan la capacidad de implementarlo" (p. 109). De manera complementaria, la MDC afirma que "no hay posibilidades de que un curriculum pueda ser concretado sin la participación y comprensión de sus propósitos y funcionamiento por parte de los prin- 
cipales protagonistas en su realización práctica a nivel pedagógico" (MINEDUC, 2016, p. 30).

Desde una perspectiva crítica, Grundy (1998) desarrolla una mirada del curriculum escolar definida por la práctica curricular situada en un momento y espacio particular. Para la autora, el curriculum es un objeto inmerso en lo humano, en la experiencia y la cultura en donde se ejecuta. Por esto, el curriculum diseñado/prescrito no tiene sentido ni existencia sin su ejecución concreta en el aula. Más aún, Grundy fundamenta:

hemos de buscar el curriculum, no en la estantería del profesor, sino que en las acciones de las personas inmersas en la educación. Por tanto, la pregunta: '¿qué es el curriculum?', se parece más a: ‘¿Qué es el fútbol?', que a: ‘¿Qué es el hidrógeno' [...] Para entender el hidrógeno solo necesitamos conocer la naturaleza del elemento mismo. Por ejemplo, no precisamos saber nada acerca del globo que están llenando de hidrógeno para entender el elemento en sí. Pero, tratándose del fútbol, tenemos que conocer un poco la sociedad en cuyo seno se practica para saber algo de la naturaleza del juego. El hidrógeno es el mismo, esté en Londres o en Sidney, pero el fútbol no. Igual ocurre con el curriculum. Ningún curriculum existe a priori. (p. 21-22).

En definitiva, independiente de la intención de quienes lo definan, el curriculum oficial tiene un efecto que escapa a la sola etapa de diseño. El imposible control de la acción educativa en el aula favorece la reproducción curricular del diseño central con variaciones inevitables, pues docentes, directivos, asistentes de la educación y estudiantes son agentes que resignifican el curriculum (Haas, Molina, Bravo \& Manghi, 2016).

A su vez, la práctica educativa se ve altamente condicionada por el curriculum oficial. Este define sus parámetros en el nivel de sistema, a través de las horas pedagógicas anuales para cada asignatura, la organización de OA en unidades como propuesta en los programas de estudio, etc. (Haas et al., 2016), para que las escuelas organicen su implementación en proyectos curriculares de centro, de aula y de planificación individualizada. 


\section{El curriculum escolar chileno}

El curriculum chileno se concretiza mediante instrumentos y componentes establecidos en la Ley General de Educación (LGE) (2009). Las BC son el principal instrumento curricular al exponer los OA mínimos para todos los establecimientos educacionales reconocidos por el Estado, buscando, primero, ofrecer una base cultural común para todo el alumnado del país y, segundo, favorecer la igualdad de oportunidades. El valor esencial de las BC en el sistema se confirma al ser el principal referente para el diseño de los Planes y Programas de Estudio, de la prueba SIMCE y de los Estándares de Aprendizaje.

A pesar de la reproducción forzosa en las escuelas de los saberes seleccionados en el nivel central, esto es, las BC, la LGE resguarda los principios de autonomía y libertad de enseñanza en la gestión curricular de las escuelas. De esta forma, las BC pueden ser complementadas con otros saberes que la comunidad puede definir:

se entrega a los establecimientos educacionales la libertad de expresar su diversidad, construyendo, a partir de ella, sus propuestas propias de acuerdo a sus necesidades y a las características de su proyecto educativo. [...] los establecimientos pueden desarrollar sus propios planes y programas o utilizar aquellos que el Ministerio de Educación pone a su disposición. Se les garantiza, además, el tiempo necesario para ejercer esta libertad, por cuanto los objetivos planteados en las Bases no utilizan la totalidad del tiempo escolar. De esta forma, se reafirma el valor de la pluralidad y la flexibilidad de opciones curriculares, y de los proyectos educativos definidos por los propios establecimientos. [...] estas Bases deben asegurar una proporción equivalente al 30\% del tiempo de trabajo escolar de libre disposición para los establecimientos que operen en el régimen de jornada escolar completa (es decir, 11 pedagógicas) para que los establecimientos dispongan libremente de él, ya sea para profundizar sobre los objetivos de las Bases o para complementarlos, según sean sus necesidades y su proyecto educativo. (MINEDUC, 2018a, pp. 18-19).

En efecto, las posibilidades de flexibilización, contextualización y complemento al curriculum oficial están garantizadas por la LGE para todas las escuelas chilenas, sean públicas o privadas (Rodríguez, 2018), a pesar de que la MDC advierte un impacto superficial de estas garan- 
tías en la práctica, a razón del reducido espacio de descentralización y de la falta de capacidades instaladas en las escuelas y el profesorado para cumplir con dichas posibilidades (MINEDUC, 2016, p. 21).

Una herramienta disponible para la flexibilización curricular corresponde a las horas de libre disposición (HLD). Estas horas pedagógicas representan una sección de tiempo lectivo a las que tienen acceso exclusivo las escuelas con Jornada Escolar Completa (JEC), y que pueden usarse para:

a) introducir en su plan de estudio asignaturas complementarias a las establecidas en las Bases Curriculares, b) incorporar una asignatura o un tema de relevancia para el proyecto educativo del establecimiento, c) incorporar una asignatura o un tema de relevancia regional o local, d) ampliar el tiempo destinado a las asignaturas obligatorias, d) dedicar el tiempo a desarrollar una asignatura o un grupo de ellas en particular, e) destinar tiempo en el horario para desarrollar algunas actividades relacionadas con los Objetivos de Aprendizaje Transversales, f) destinar tiempo a actividades deportivas, artísticas o a nivelación de alumnos y alumnas. (MINEDUC, 2012, citado en Castillo \& Martínez, 2017, p.10).

En lo concreto, más allá de la normativa, la posibilidad de flexibilización, contextualización y complemento del curriculum oficial no es total para el sistema escolar, pues se reserva exclusivamente a los establecimientos con JEC. A las escuelas sin JEC les queda la posibilidad de una autonomía un tanto reducida de elaborar sus propios planes y programas, los que deben pasar por un proceso de aprobación oficial que incluso puede poner en riesgo el "mínimo común" expuesto por las $\mathrm{BC}$, y el principio de igualdad de oportunidades para el estudiantado.

Ahora bien, en el caso de los establecimientos con JEC y el uso efectivo de sus HLD, la investigación de Castillo y Martínez (2017) detectó que la mayoría 2.483 instituciones escolares chilenas estudiadas destina las HLD a la profundización de aprendizaje en Lenguaje y Matemática (71\% y $64 \%$, respectivamente), a la enseñanza de un idioma $(63 \%)^{2}$, al desarro-

2 Podría explicarse porque entre $1^{\circ}$ y $4^{\circ}$ básico la asignatura de Inglés no está dentro del Plan de Estudio, por lo que su enseñanza depende de las HLD. 
llo de "otras actividades" (62\%) ${ }^{3}$, a actividades deportivas (58\%), a la formación artística (57\%), y a la orientación (51\%). Menormente, se utiliza el tiempo en otras asignaturas ligadas a las ciencias naturales y sociales (34\% y 21\%), a la preparación de la PSU (23\%, solo en educación media), y a los consejos de curso (21\%), entre otros. Es posible interpretar que, por un lado, las posibilidades de complemento curricular se condicionan por las necesidades operativas de los propios establecimientos, por ejemplo, captación de matrículas de estudiantes, y, por otro, que estas escuelas suelen usar los resultados SIMCE de Lenguaje y Matemática, y la enseñanza del inglés, como principales recursos propagandísticos, por lo que destinan las HLD al refuerzo de estos aprendizajes.

El mismo trabajo expuso que la distribución de las HLD se sustenta, de mayor a menor medida, en el ideario de su Proyecto Educativo Institucional ( $60 \%$ de los establecimientos), los intereses del alumnado (58\%), el sello institucional (53\%) y la formación integral del alumnado (50\%), entre otros. Finalmente, se recogió que la dirección del establecimiento es la que define el uso de las HLD (93,9\% de los encuestados), seguido por las jefaturas de UTP $(81,5 \%)$ y el profesorado $(78,4 \%)$. La participación de otros integrantes de la comunidad educativa es muy menor $(29,2 \%)$, lo que podría demostrar una débil pertinencia del complemento curricular de la escuela, al apartar de la decisión a parte importante de esta.

En la misma línea, Huepe y Valenzuela (2008, citado en Castillo \& Martínez, 2017) identifican cinco lógicas sobre el uso de las HLD en Chile, predominando una naturaleza remedial al curriculum oficial:

I. Lógica compensatoria: Se refuerzan contenidos débiles que no alcanzaron a ser cubiertos por falta de tiempo o por la presencia de estudiantes con bajo capital cultural, o para elevar los resultados en la prueba SIMCE.

II. Lógica proyectiva: Se apoya la preparación de estudiantes para la Prueba de Selección Universitaria.

3 Como "otras actividades" se entiende aquellas que exceden a las asignaturas principales del curriculum oficial, las actividades deportivas, la formación artística, la preparación de pruebas externas, el consejo de curso, la formación ciudadana y de orientación. 
III. Lógica formativa: Se apoya la formación de habilidades artísticas y deportivas.

IV. Lógica contingente: Uso sin lógica subyacente, ni objetivos de aprendizaje definidos.

V. Lógica académica: Se aumentan las horas de asignaturas como Ciencias, Lenguaje y Matemática.

Por otra parte, en el curriculum chileno los planes de estudio informan el tiempo mínimo anual requerido para cada asignatura y curso. Resulta esta una definición siempre controversial, dado que las unidades de tiempo atribuidas a una disciplina y contenido podrían expresar el estatus social de cada campo del conocimiento y el tipo de relación entre dicho campos, con efectos sociales no despreciables (Bernstein, 1985).

A su vez, los programas de estudio proponen una organización temporal de los OA también según asignatura y curso. Los programas, bajo una lógica de flexibilidad, para que se adapten a cada realidad, sugieren una secuencia de OA y una estimación de horas pedagógicas para su logro. Además, ofrecen ejemplos de actividades de aprendizaje y de evaluación, e indicadores de logro. Tanto planes como programas tienen un carácter obligatorio; sin embargo, los establecimientos pueden elaborar y aplicar los propios, cumpliendo con el tiempo mínimo determinado para las asignaturas de Lenguaje y Comunicación y Matemática, y la autorización oficial (MINEDUC, 2018a).

A los instrumentos curriculares se unen los componentes: Objetivos de Aprendizaje y Objetivos de Aprendizaje Transversales (OAT). Los OA definen los aprendizajes esperables en una asignatura al final de cada año escolar, integrando en su formulación: conocimientos (conceptos, redes de conceptos e información sobre hechos, procesos, procedimientos y operaciones), habilidades (capacidades para realizar tareas y solución problemas) y actitudes (disposiciones para responder frente a objetos, ideas o personas). Los OAT expresan aprendizajes referidos al desarrollo personal de los estudiantes y a su conducta moral y social, destinados a ser alcanzados al final de un nivel $\left(1^{\circ}\right.$ a $6^{\circ}$ de educación básica, y $7^{\circ}$ básico a $\mathrm{IV}^{\circ}$ medio), considerando todas las asignaturas y experiencias en el establecimiento, incluido lo extraescolar (MINEDUC, 2018a). 
$\mathrm{Al}$ analizar la función de los OA en el actual curriculum, Oliva (2017) es crítico y plantea que escasamente se desmarcan del curriculum anterior, es decir, de la racionalidad técnica; más bien la "refinan". Los OA vigentes mantienen un carácter eficiente, conductista y psicométrico, sostiene Oliva. También, a razón de dicha racionalidad, que apunta al control "silencioso" de la práctica educativa (Pascual, 1998), se observa un curriculum sobrecargado por un listado cuantioso de objetivos anuales, abundancia que da poco margen a la incorporación de saberes del contexto de cada escuela (MINEDUC, 2016; Rodríguez, 2018), desembocando en una cobertura curricular incompleta en gran parte de los establecimientos (Contreras, 2014; MINEDUC, 2015). Pareciese que el control educativo para la reproducción del curriculum oficial se desarrolla mediante su propia saturación, con el fin de copar los espacios de autonomía de los establecimientos: a mayor volumen curricular central, mayor control de la divergencia. En este contexto surge el concepto de "cobertura curricular", pues problemas con esta suponen un contratiempo para la aplicación del curriculum oficial y su posterior contextualización.

Conceptualmente, la cobertura curricular guarda estrecha relación con la densidad curricular y su desarrollo, y concierne a la cantidad o porcentaje de metas formativas que alcanzan a ser enseñadas respecto de las planificadas, entendiéndose una relación entre la cobertura curricular de un establecimiento sobre el curriculum oficial y la igualdad de oportunidades del estudiantado. Sin embargo, se reconoce que, en la misma práctica, el profesorado, con un tiempo pedagógico limitado, debe decidir entre enseñar menos contenidos a favor de mayor profundidad, o enseñar más pero superficialmente (Rodríguez, Castillo \& Saavedra, 2015).

El estudiantado de grupos ancestrales rurales vive casos extremos de baja cobertura. Se ha demostrado una brecha significativamente mayor entre curriculum prescrito y curriculum enseñado, presumiblemente por el desapego territorial a las BC y por las bajas expectativas de aprendizaje de la escuela que atiende a este alumnado (Arias \& Quintriqueo, 2020).

Por lo demás, existe evidencia suficiente respecto de la baja cobertura del curriculum escolar chileno en las escuelas. Rodríguez et al. (2015), fundados en la opinión de ocho mil docentes de Matemática, recogió que cerca del $36 \%$ de los contenidos de $8^{\circ}$ básico no son enseñados con suficiente profundidad. 
A su vez, el Centro de Estudios Mineduc (2013) consultó a más de dos mil docentes de Lenguaje y Matemática de educación media, evidenciando una cobertura disminuida del curriculum en sus asignaturas: $82 \%$ y $73 \%$, respectivamente. En la sección cualitativa de la misma investigación, se consignó que en ninguno de los doce establecimientos participantes se logró enseñar la totalidad del curriculum. Como matiz, los datos reportaron que las escuelas privadas sin subvención estatal presentan una cobertura curricular superior a la de las públicas.

Mas evidencias se exponen en el Estudio de implementación curricular en Enseñanza Básica (MINEDUC, 2015). Este concluyó que, en promedio, en ninguno de los cursos $\left(1^{\circ}\right.$ y $6^{\circ}$ básico) y asignaturas (nueve) del profesorado consultado (más de diez mil), los OA de las BC son cubiertos totalmente. La tabla 1 expone estos resultados pormenorizadamente:

Tabla 1

Horas pedagógicas dedicadas a cada asignatura y cobertura curricular

\begin{tabular}{|c|c|c|c|c|c|c|c|}
\hline Asignatura & Categoría Nivel & $1^{\circ}$ & $2^{\circ}$ & $3^{\circ}$ & $4^{\circ}$ & $5^{\circ}$ & $6^{\circ}$ \\
\hline \multirow[t]{2}{*}{ Lenguaje } & $\begin{array}{l}\text { Horas } \\
\text { pedagógicas } \\
\text { dedicadas a la } \\
\text { asignatura }\end{array}$ & 8 & 7,5 & 8,1 & 7,9 & 6,7 & 6,5 \\
\hline & $\begin{array}{l}\text { Cobertura de OA } \\
\text { en el año escolar }\end{array}$ & $92,9 \%$ & $94,1 \%$ & $92,2 \%$ & $93,6 \%$ & $91,1 \%$ & $92,0 \%$ \\
\hline \multirow[t]{2}{*}{ Inglés } & $\begin{array}{l}\text { Horas } \\
\text { pedagógicas } \\
\text { dedicadas a la } \\
\text { asignatura }\end{array}$ & & & & & 3,5 & 3,4 \\
\hline & $\begin{array}{l}\text { Cobertura de OA } \\
\text { en el año escolar }\end{array}$ & & & & & $90,2 \%$ & $88,5 \%$ \\
\hline \multirow[t]{2}{*}{ Matemática } & $\begin{array}{l}\text { Horas } \\
\text { pedagógicas } \\
\text { dedicadas a la } \\
\text { asignatura }\end{array}$ & 6,4 & 6,5 & 6,6 & 6,7 & 6,4 & 6,4 \\
\hline & $\begin{array}{l}\text { Cobertura de OA } \\
\text { en el año escolar }\end{array}$ & $95,6 \%$ & $89 \%$ & $90,5 \%$ & $91,96 \%$ & $85,6 \%$ & $88,5 \%$ \\
\hline \multirow[t]{2}{*}{ Historia } & $\begin{array}{l}\text { Horas } \\
\text { pedagógicas } \\
\text { dedicadas a la } \\
\text { asignatura }\end{array}$ & 3,1 & 3,1 & 3,2 & 3,2 & 4,1 & 4,1 \\
\hline & $\begin{array}{l}\text { Cobertura de OA } \\
\text { en el año escolar }\end{array}$ & $96,3 \%$ & $94,7 \%$ & $95,2 \%$ & $91,1 \%$ & $83,3 \%$ & $85,0 \%$ \\
\hline
\end{tabular}




\begin{tabular}{|c|c|c|c|c|c|c|c|}
\hline \multirow[t]{2}{*}{$\begin{array}{l}\text { Ciencias } \\
\text { Naturales }\end{array}$} & $\begin{array}{l}\text { Horas } \\
\text { pedagógicas } \\
\text { dedicadas a la } \\
\text { asignatura }\end{array}$ & 3,1 & 3,1 & 3,2 & 3,2 & 3,9 & 3,9 \\
\hline & $\begin{array}{l}\text { Cobertura de OA } \\
\text { en el año escolar }\end{array}$ & $95,4 \%$ & $94,8 \%$ & $95,2 \%$ & $92,9 \%$ & $94,8 \%$ & $93,4 \%$ \\
\hline \multirow[t]{2}{*}{$\begin{array}{l}\text { Artes } \\
\text { Visuales }\end{array}$} & $\begin{array}{l}\text { Horas } \\
\text { pedagógicas } \\
\text { dedicadas a la } \\
\text { asignatura }\end{array}$ & 1,9 & 2 & 2 & 2 & 1,9 & 1,9 \\
\hline & $\begin{array}{l}\text { Cobertura de OA } \\
\text { en el año escolar }\end{array}$ & $92 \%$ & $92,3 \%$ & $91,7 \%$ & $90,6 \%$ & $89,9 \%$ & $88,0 \%$ \\
\hline \multirow[t]{2}{*}{ Música } & $\begin{array}{l}\text { Horas } \\
\text { pedagógicas } \\
\text { dedicadas a la } \\
\text { asignatura }\end{array}$ & 1,9 & 1,9 & 2 & 2 & 1,8 & 1,8 \\
\hline & $\begin{array}{l}\text { Cobertura de OA } \\
\text { en el año escolar }\end{array}$ & $91,7 \%$ & $90,1 \%$ & $88,1 \%$ & $87,1 \%$ & $83,1 \%$ & $82,9 \%$ \\
\hline \multirow[t]{2}{*}{$\begin{array}{l}\text { Educación } \\
\text { Física }\end{array}$} & $\begin{array}{l}\text { Horas } \\
\text { pedagógicas } \\
\text { dedicadas a la } \\
\text { asignatura }\end{array}$ & 3,3 & 3,4 & 3,7 & 3,7 & 2,6 & 2,5 \\
\hline & $\begin{array}{l}\text { Cobertura de OA } \\
\text { en el año escolar }\end{array}$ & $92,0 \%$ & $90,0 \%$ & $90,0 \%$ & $90,6 \%$ & $88,3 \%$ & $90,7 \%$ \\
\hline \multirow[t]{2}{*}{ Tecnología } & $\begin{array}{l}\text { Horas } \\
\text { pedagógicas } \\
\text { dedicadas a la } \\
\text { asignatura }\end{array}$ & 1,3 & 1,4 & 1,3 & 1,4 & 1,4 & 1,5 \\
\hline & $\begin{array}{l}\text { Cobertura de OA } \\
\text { en el año escolar }\end{array}$ & $83,7 \%$ & $83,2 \%$ & $84,0 \%$ & $84,4 \%$ & $82,8 \%$ & $83,2 \%$ \\
\hline
\end{tabular}

Nota: Elaborado a partir de resultados del Estudio de implementación curricular en Enseñanza Básica (MINEDUC, 2015).

Por su parte, entendiendo que el éxito de un curriculum oficial (que los OA sean cubiertos completamente en el aula, en profundidad y con aprendizajes adecuados) está en manos de sus mediadores en la escuela, directivos y docentes, los resultados del Estudio de exploración y análisis de los procesos de implementación curricular en el sistema educacional chileno (MINEDUC, 2018b) son esclarecedores, al demostrar las complicaciones recurrentes en la concreción curricular en la escuela y en el aula. Sobre el profesorado, algunas conclusiones son:

- Desconoce las BC o las confunden con los Planes y Programas de Estudio. Hay un predominio en el uso de los Planes y Programas de estudio por sobre las BC. 
- Identifica a los Programas de Estudio como "el curriculum", ignorando otros instrumentos, medios y materiales como las BC, los Estándares de Aprendizaje, los textos escolares, entre otros.

- Tiende a atribuir mayor nivel de prescripción a los Programas de Estudio que el que efectivamente tienen.

- Otorga un carácter obligatorio a elementos que solo son sugeridos.

- Valora a los OA como organizadores del curriculum, pero presenta una comprensión desarticulada de sus componentes (conocimientos, habilidades y actitudes): "algunos docentes declaran la imposibilidad de desarrollar habilidades por falta de tiempo para cubrir los contenidos, o la incongruencia del currículum versus las exigencias de las evaluaciones estandarizadas que miden contenidos y no habilidades" (p. 65).

- Junto a los directivos, realizan el seguimiento curricular respecto de la "cobertura de contenidos", pero no de "cobertura de aprendizajes". Conceptualmente contenidos y aprendizajes son distintos.

- Muestra escasos procesos de contextualización curricular, salvo adaptaciones a estudiantes con Necesidades Educativas Especiales (NEE).

- Concede baja credibilidad a la política curricular.

En la misma línea, la desconfianza de docentes y equipos directivos sobre el curriculum oficial se nutre de las contradicciones observadas entre la política curricular nacional y el sistema educativo. Por ejemplo, se advierte una perspectiva curricular praxiológica que domina el diseño prescriptivo del curriculum nacional, pero que colisiona con políticas educativas y evaluativas centradas en los resultados atribuidos principalmente al profesorado y a la escuela (perspectiva técnica). Esto repercute en la deliberación, decisión y valoración de los establecimientos sobre el curriculum oficial (Ossandón \& Pinto, 2018).

Otra explicación a las confusiones y molestias del profesorado sobre el curriculum oficial está en los procesos curriculares derivados de la aprobación de la LGE en 2009. Durante la década 2010-2019, en el sistema chileno existió una superposición de dos currículos oficiales (Ajuste curricular 2009 y Reforma curricular 2012). Esta contradicción repercutió en la ausencia de programas de estudio en algunas asignaturas, desajustes en la producción de los textos de estudio del alumnado, postergaciones intempestivas y cambios en los cronogramas de 
aplicación del ajuste curricular, convivencia de instrumentos y componentes curriculares de diferente origen legal ${ }^{4}$; todo esto redundando en informaciones confusas para escuelas y profesorado (Espinoza, 2016). Tales hechos han perpetuado la tensión entre profesorado, escuela y curriculum oficial, arriesgando su apropiación correcta (Cox, 2018).

\section{Curriculum escolar chileno para la enseñanza en pandemia: priorización curricular}

La siguiente revisión se realiza en función del documento oficial Orientaciones para la Implementación de la Priorización Curricular en Forma Remota y Presencial (MINEDUC, 2020c), el que expone nuevas características de la propuesta de priorización.

Primeramente se plantea que la priorización para educación prescolar/inicial, y primer y segundo ciclo escolar es exclusivamente una "herramienta de apoyo curricular", y no un nuevo curriculum. Es una propuesta transitoria, no vinculante, con eficacia hasta 2021, pues las $\mathrm{BC}$ se encuentran plenamente vigentes. En segundo orden, se sostiene que la priorización entrega mayor protagonismo curricular al equipo directivo y docentes, dado que estos deberán diseñar y ajustar plan de estudio, metodologías y evaluaciones a sus propias condiciones. En el documento es notable la ausencia de la comunidad escolar como grupo de agentes intervinientes, arriesgando el propio éxito de la priorización,

Sobre las asignaturas, Orientación y Tecnología no presentan una propuesta de priorización como tal, sino que se abordarían transversalmente en las demás asignaturas, "dada su relevancia en el contexto que estamos viviendo" (MINEDUC, 2020c, p. 2). En el caso de Religión se sugiere su integración al plan de estudio ajustado, "pues [esta] impacta en una dimensión de los estudiantes que es de vital importancia" (MINEDUC, 2020c, p. 2).

Por otro lado, la priorización no solo se redujo a la selección de un conjunto de $\mathrm{OA}$ esenciales desde las $\mathrm{BC}$, sino que también implicó una clasificación interna de dicho conjunto: OA de Nivel P1 (primer nivel) y OA de Nivel P2 (segundo nivel).

4 Ejemplos de oposiciones: Marco Curricular y Bases Curriculares; Objetivos Fundamentales y Contenidos Mínimos Obligatorios, y Objetivos de Aprendizaje; Sectores y Subsectores de Aprendizaje, y Asignatura. 
El Nivel P1 de OA representa “un primer mínimo”, es decir, una escala más alta de prioridad de la enseñanza, mientras que el Nivel P2 posee un carácter suplementario; no obstante, se advierte en el documento que "si el tiempo de educación presencial se ve extremadamente reducido, se recomienda dedicarse al máximo posible al Nivel P1, quedando sin efecto el Nivel P2" (MINEDUC, 2020c, p. 3).

Respecto de la adecuación del Plan de Estudio, MINEDUC sugiere lo indicado en la tabla 2, atendiendo a la flexibilidad que proporciona la priorización y a las medidas sanitarias vigentes.

Tabla 2.

Posibilidad de ajuste al Plan de Estudios según escenarios de retorno a clases presenciales

\begin{tabular}{cc}
\hline $\begin{array}{l}\text { Escenarios posibles al momento } \\
\text { del retorno a clases en año 2020, en } \\
\text { horas semanales }\end{array}$ & $\begin{array}{l}\mathrm{N}^{\circ} \text { de asignaturas o módulos } \\
\text { posibles de incluir en el Plan de } \\
\text { Estudios de todas las asignaturas }\end{array}$ \\
\hline 34 a 42 & 6 asignaturas o más \\
\hline 31 a 33 & 5 a 6 asignaturas \\
\hline 21 a 30 & 4 a 5 asignaturas \\
\hline 16 a 20 & 3 a 4 asignaturas \\
\hline 6 a 15 & 2 a 3 asignaturas \\
\hline
\end{tabular}

Notas: (1) Tabla reproducida fielmente de MINEDUC (2020c).

(2) Los escenarios posibles se supeditan a las medidas sanitarias.

(3) Es una sugerencia que afecta a todos los cursos, tipos de jornada y establecimientos que imparten la asignatura de Lengua Indígena.

No obstante esta sugerencia, cada establecimiento podrá jerarquizar autónomamente sus asignaturas, aunque MINEDUC (2020c) prescribe una excepción: "En todos los escenarios, el Plan de Estudios debe incluir las asignaturas de Lenguaje y Comunicación (Lengua y Literatura) y Matemática, con las horas asignadas en el Plan de Estudios vigente. Además, se recomienda agregar una asignatura del ámbito artístico" (p. 7). A la vez, se recomienda otras acciones:

- Si la jornada ha disminuido sustantivamente, profundizar en menos asignaturas.

- Planificar horarios diferidos por grupos de estudiantes.

- En $1^{\circ}$ y $2^{\circ}$ básico hacer prevalecer la lectura comprensiva, integrándose con otras asignaturas, por ejemplo, "incluir las lecturas de los 
Textos Escolares de Ciencias e Historia como parte de la lectura en voz alta y compartida, utilizar el arte para expresar los significados de los textos, emplear la música para aprender palabras nuevas y poesía, integrar la tecnología para complementar el aprendizaje de la lectura" (Id., p. 7).

- Incluir en la planificación de aula proyectos que integren diversas asignaturas, por ejemplo, STEAM (Ciencias, Tecnología, Matemática y Arte).

Por otro lado, el escenario de la priorización curricular atiende dos realidades educativas: presencial y remota. Para MINEDUC, la modalidad presencial implica para la escuela recoger información acerca de las posibilidades de asistencia escolar, la participación mixta (presencial y remota) y el apoyo a los apoderados. Para la planificación en esta modalidad se propone:

- Diseñar clases para todo el estudiantado, en especial para el que requiere más apoyo. Para estudiantes con NEE y en los "cursos críticos" ( $1^{\circ}$ a $2^{\circ}$ básico, $8^{\circ}$ básico a ${ }^{\circ}$ medio, y $\mathrm{IV}^{\circ}$ medio), planificar colaborativamente entre los docentes.

- Planificar espacios para ofrecer contención socioemocional, en especial, durante Orientación.

- Integrar los aprendizajes de Tecnología (sobre todo TIC) como herramienta de apoyo a otras asignaturas.

- Utilizar fichas pedagógicas y recursos alineados a los textos escolares disponibles en el portal Aprendo en Línea.

- Permitir que el alumnado lleve el texto escolar al hogar.

A su vez, para la enseñanza en modalidad remota o virtual, MINEDUC recomienda, por ejemplo:

- Diagnosticar la situación del estudiantado sobre su conectividad, habilidades digitales, apoyo de apoderados y estado socioemocional.

- Incentivar el uso de plataformas digitales (Aprendo en línea) y las que la escuela defina.

- Planificar estrategias de comunicación con estudiantes para guiarlos en el aprendizaje en el hogar, y para informar y apoyar educativamente a las familias. 
En este trabajo se revisará la priorización curricular dispuesta solo para el primer ciclo ( $1^{\circ}$ a $6^{\circ}$ básico), en forma general. Un análisis cualitativo y pormenorizado requerirá de mayor espacio.

La tabla 3 expresa cuantitativamente la relación entre los OA de las $\mathrm{BC}$ vigentes y los OA priorizados, con el fin de estimar la cobertura curricular del segundo respecto del primero, distinguiéndose además los OA priorizados Nivel P1 y Nivel P2.

Tabla 3.

Resumen cantidad OA según BC y OA priorizados en primer ciclo ( $1^{\circ}$ a $6^{\circ}$ básico)

\begin{tabular}{|c|c|c|c|c|c|c|c|}
\hline Asignatura & Curriculum & $\begin{array}{c}\text { Cantidad } \\
\text { OA } 1^{\circ}\end{array}$ & $\begin{array}{c}\text { Cantidad } \\
\text { OA } 2^{\circ}\end{array}$ & $\begin{array}{c}\text { Cantidad } \\
\text { OA } 3^{\circ}\end{array}$ & $\begin{array}{c}\text { Cantidad } \\
\text { OA } 4^{\circ}\end{array}$ & $\begin{array}{l}\text { Cantidad } \\
\text { OA } 5^{\circ}\end{array}$ & $\begin{array}{c}\text { Cantidad } \\
\text { OA } 6^{\circ}\end{array}$ \\
\hline \multirow{4}{*}{$\begin{array}{l}\text { Lenguaje y } \\
\text { Comunicación }\end{array}$} & $\mathrm{BC}$ & 26 & 30 & 31 & 30 & 30 & 31 \\
\hline & Priorización & $5(\mathrm{P} 1)$ & $7(\mathrm{P} 1)$ & $7(\mathrm{P} 1)$ & $7(\mathrm{P} 1)$ & $8(\mathrm{P} 1)$ & $8(\mathrm{P} 1)$ \\
\hline & curricular y & $2(\mathrm{P} 2)$ & $3(\mathrm{P} 2)$ & $2(\mathrm{P} 2)$ & $2(\mathrm{P} 2)$ & $2(\mathrm{P} 2)$ & $2(\mathrm{P} 2)$ \\
\hline & $\begin{array}{l}\text { \% cobertura } \\
\text { curricular }\end{array}$ & $27 \%$ & $33 \%$ & $29 \%$ & $30 \%$ & $32 \%$ & $33 \%$ \\
\hline \multirow[t]{4}{*}{ Matemática } & $\mathrm{BC}$ & 20 & 22 & 26 & 27 & 27 & 24 \\
\hline & Priorización & $4(\mathrm{P} 1)$ & $5(\mathrm{P} 1)$ & $7(\mathrm{P} 1), 7$ & $7(\mathrm{P} 1)$ & 7 (P1) & $6(\mathrm{P} 1)$ \\
\hline & curricular y & $6(\mathrm{P} 2)$ & $7(\mathrm{P} 2)$ & (P2) & $7(\mathrm{P} 2)$ & $8(\mathrm{P} 2)$ & $8(\mathrm{P} 2)$ \\
\hline & $\begin{array}{l}\text { \% cobertura } \\
\text { curricular }\end{array}$ & $50 \%$ & $55 \%$ & $54 \%$ & $52 \%$ & $56 \%$ & $58 \%$ \\
\hline \multirow[t]{4}{*}{ Inglés } & $\mathrm{BC}$ & & & & & 16 & 16 \\
\hline & Priorización & & & & & $4(\mathrm{P} 1)$ & $4(\mathrm{P} 1)$ \\
\hline & curricular y & & & & & $1(\mathrm{P} 2)$ & $1(\mathrm{P} 2)$ \\
\hline & $\begin{array}{l}\text { \% cobertura } \\
\text { curricular }\end{array}$ & & & & & $31 \%$ & $31 \%$ \\
\hline \multirow{4}{*}{$\begin{array}{l}\text { Historia y } \\
\text { Geografía } \\
\text { y Ciencias } \\
\text { Sociales }\end{array}$} & $\mathrm{BC}$ & 15 & 16 & 16 & 18 & 22 & 26 \\
\hline & Priorización & $5(\mathrm{P} 1)$ & $5(\mathrm{P} 1)$ & $5(\mathrm{P} 1)$ & $5(\mathrm{P} 1)$ & $5(\mathrm{P} 1)$ & $6(\mathrm{P} 1)$ \\
\hline & curricular y & $1(\mathrm{P} 2)$ & $1(\mathrm{P} 2)$ & $1(\mathrm{P} 2)$ & $1(\mathrm{P} 2)$ & $1(\mathrm{P} 2)$ & $2(\mathrm{P} 2)$ \\
\hline & $\begin{array}{l}\text { \% cobertura } \\
\text { curricular }\end{array}$ & $40 \%$ & $38 \%$ & $38 \%$ & $33 \%$ & $27 \%$ & $31 \%$ \\
\hline \multirow{4}{*}{$\begin{array}{l}\text { Ciencias } \\
\text { Naturales }\end{array}$} & $\mathrm{BC}$ & 12 & 14 & 13 & 17 & 14 & 18 \\
\hline & Priorización & $4(\mathrm{P} 1)$ & $4(\mathrm{P} 1)$ & $4(\mathrm{P} 1)$ & $5(\mathrm{P} 1)$ & $3(\mathrm{P} 1)$ & $5(\mathrm{P} 1)$ \\
\hline & curricular y & $2(\mathrm{P} 2)$ & $2(\mathrm{P} 2)$ & 3 (P2) & 3 (P2) & 3 (P2) & $5(\mathrm{P} 2)$ \\
\hline & $\begin{array}{l}\text { \% cobertura } \\
\text { curricular }\end{array}$ & $50 \%$ & $43 \%$ & $54 \%$ & $47 \%$ & $43 \%$ & $56 \%$ \\
\hline \multirow[t]{4}{*}{ Artes Visuales } & $\mathrm{BC}$ & 5 & 5 & 5 & 5 & 5 & 5 \\
\hline & Priorización & $1(\mathrm{P} 1)$ & $1(\mathrm{P} 1)$ & $1(\mathrm{P} 1)$ & $1(\mathrm{P} 1)$ & $1(\mathrm{P} 1)$ & $1(\mathrm{P} 1)$ \\
\hline & curricular y & $2(\mathrm{P} 2)$ & $2(\mathrm{P} 2)$ & $2(\mathrm{P} 2)$ & $2(\mathrm{P} 2)$ & $2(\mathrm{P} 2)$ & $2(\mathrm{P} 2)$ \\
\hline & $\begin{array}{l}\text { \% cobertura } \\
\text { curricular }\end{array}$ & $60 \%$ & $60 \%$ & $60 \%$ & $60 \%$ & $60 \%$ & $60 \%$ \\
\hline \multirow{4}{*}{$\begin{array}{l}\text { Lengua y } \\
\text { cultura de } \\
\text { los pueblos } \\
\text { originarios } \\
\text { ancestrales }\end{array}$} & $\mathrm{BC}$ & 11 & 12 & & & & \\
\hline & Priorización & $4(\mathrm{P} 1)$ & $4(\mathrm{P} 1)$ & & & & \\
\hline & curricular y & $2(\mathrm{P} 2)$ & $2(\mathrm{P} 2)$ & & & & \\
\hline & $\begin{array}{l}\text { \% cobertura } \\
\text { curricular }\end{array}$ & $55 \%$ & $50 \%$ & & & & \\
\hline
\end{tabular}




\begin{tabular}{|c|c|c|c|c|c|c|c|}
\hline \multirow{4}{*}{$\begin{array}{l}\text { Lengua } \\
\text { Indígena }\end{array}$} & \multirow{4}{*}{$\begin{array}{l}\text { BC } \\
\text { Priorización } \\
\text { curricular y } \\
\text { \% cobertura } \\
\text { curricular }\end{array}$} & & & \multicolumn{2}{|c|}{$11^{5}$} & \multirow{2}{*}{$\begin{array}{c}9 \\
2(\mathrm{P} 1)\end{array}$} & \multirow{2}{*}{$\frac{10}{2(\mathrm{P} 1)}$} \\
\hline & & & & $2(\mathrm{P} 1)$ & $2(\mathrm{P} 1)$ & & \\
\hline & & & & $2(\mathrm{P} 2)$ & $2(\mathrm{P} 2)$ & $2(\mathrm{P} 2)$ & $2(\mathrm{P} 2)$ \\
\hline & & & & & & $44 \%$ & $40 \%$ \\
\hline \multirow{4}{*}{$\begin{array}{l}\text { Educación } \\
\text { Física y Salud }\end{array}$} & $\mathrm{BC}$ & 11 & 11 & 11 & 11 & 11 & 11 \\
\hline & Priorización & $3(\mathrm{P} 1)$ & $3(\mathrm{P} 1)$ & $3(\mathrm{P} 1)$ & $3(\mathrm{P} 1)$ & $3(\mathrm{P} 1)$ & $3(\mathrm{P} 1)$ \\
\hline & curricular y & $1(\mathrm{P} 2)$ & $1(\mathrm{P} 2)$ & $1(\mathrm{P} 2)$ & $1(\mathrm{P} 2)$ & 1 (P2) & $1(\mathrm{P} 2)$ \\
\hline & $\begin{array}{l}\text { \% cobertura } \\
\text { curricular }\end{array}$ & $36 \%$ & $36 \%$ & $36 \%$ & $36 \%$ & $36 \%$ & $36 \%$ \\
\hline \multirow[t]{4}{*}{ Música } & $\mathrm{BC}$ & 7 & 7 & 8 & 8 & 8 & 8 \\
\hline & Priorización & $1(\mathrm{P} 1)$ & $1(\mathrm{P} 1)$ & $1(\mathrm{P} 1)$ & $1(\mathrm{P} 1)$ & $1(\mathrm{P} 1)$ & $1(\mathrm{P} 1)$ \\
\hline & curricular y & 2 (P2) & $2(\mathrm{P} 2)$ & $2(\mathrm{P} 2)$ & $2(\mathrm{P} 2)$ & 2 (P2) & $2(\mathrm{P} 2)$ \\
\hline & $\begin{array}{l}\% \text { cobertura } \\
\text { curricular }\end{array}$ & $43 \%$ & $43 \%$ & $38 \%$ & $38 \%$ & $38 \%$ & $38 \%$ \\
\hline
\end{tabular}

Nota: Elaboración a partir de MINEDUC (s/f)

Significado de siglas: $\mathrm{P} 1$ = OA de primer nivel; $\mathrm{P} 2$ = OA de segundo nivel.

De lo visto, en primer lugar la cobertura del curriculum priorizado en cada asignatura es mucho menor que la que declaró cumplir el profesorado en un año escolar normal en el Estudio de implementación curricular en Enseñanza Básica (MINEDUC, 2015).

En Lenguaje y Comunicación, "accidentalmente", los OA priorizados revelan la gran cantidad de OA presentes en las BC (hasta treinta y un $\mathrm{OA}$ en $2^{\circ}$ y $6^{\circ}$ básico). Tal cosa explicaría el bajo porcentaje de cobertura curricular del curriculum priorizado en la asignatura respecto de las BC, confirmando las serias dificultades en el cumplimiento de las metas formativas en un periodo normal, algo demostrado en los estudios anteriormente descritos.

Si bien en Matemática la cantidad de $\mathrm{OA}$ en $\mathrm{BC}$ es relativamente menor a Lenguaje (hasta veintisiete $\mathrm{OA}$ en $4^{\circ}$ y $5^{\circ}$ básico), la priorización considera más del $50 \%$ de cobertura en la mayoría de los cursos, aunque este porcentaje está abultado por los OA prioritarios de segundo nivel (P2), es decir, los suplementarios. En efecto, en la suma de OA de Nivel P1, Lenguaje supera a Matemática (42 a 36).

Por otro lado, tanto en Artes Visuales como en Música el número de OA de Nivel P1 es menor (1), convirtiéndose en las más bajas representaciones entre todas las asignaturas. Finalmente, Tecnología y

$5 \quad$ Planteados como “Objetivos Fundamentales”, al igual que en $5^{\circ}$ y $6^{\circ}$ básico. 
Orientación pierden visibilidad en el Plan de Estudio, sacrificando la certificación de aprendizajes —evaluación sumativa — en Tecnología.

Resulta notable como la discreta presencia de los aprendizajes de estas cuatro asignaturas, más Educación Física y Salud, en el curriculum priorizado, contraviene las necesidades de la coyuntura pandémica expresadas por la CEPAL-UNESCO (2020): "la solidaridad, el aprendizaje autónomo, el cuidado propio y de otros, las competencias socioemocionales, la salud y la resiliencia, entre otros" (p. 4).

En otro análisis, la tabla 4 muestra la representación de los ejes de cada asignatura en el curriculum priorizado, de acuerdo a los OA de Nivel P1 (aprendizajes esenciales):

Tabla 4.

Cantidad de OA de primer nivel por ejes de asignatura en el curriculum priorizado

\begin{tabular}{|c|c|c|c|c|c|c|c|}
\hline Asignatura & Ejes & $\begin{array}{c}1^{\circ} \\
\text { básico }\end{array}$ & $\begin{array}{c}2^{\circ} \\
\text { básico }\end{array}$ & $\begin{array}{c}3^{\circ} \\
\text { básico }\end{array}$ & $\begin{array}{c}4^{\circ} \\
\text { básico }\end{array}$ & $\begin{array}{c}5^{\circ} \\
\text { básico }\end{array}$ & $\begin{array}{c}6^{\circ} \\
\text { básico }\end{array}$ \\
\hline \multirow{3}{*}{$\begin{array}{l}\text { Lenguaje y } \\
\text { Comunicación }\end{array}$} & Lectura & 2 & 3 & 3 & 3 & 4 & 4 \\
\hline & Escritura & 1 & 2 & 2 & 2 & 3 & 3 \\
\hline & $\begin{array}{l}\text { Comunicación } \\
\text { Oral }\end{array}$ & 2 & 2 & 2 & 2 & 1 & 1 \\
\hline \multirow{5}{*}{ Matemática } & $\begin{array}{l}\text { Números y } \\
\text { operaciones }\end{array}$ & 1 & 2 & 3 & 3 & 3 & 2 \\
\hline & Patrones y álgebra & 1 & 1 & 1 & 1 & 1 & 1 \\
\hline & Geometría & 1 & 1 & 1 & 1 & 1 & 1 \\
\hline & Medición & 1 & 1 & 1 & 1 & 1 & 1 \\
\hline & $\begin{array}{l}\text { Datos y } \\
\text { probabilidades }\end{array}$ & 0 & 0 & 1 & 1 & 1 & 1 \\
\hline \multirow[t]{4}{*}{ Inglés } & $\begin{array}{l}\text { Comprensión } \\
\text { auditiva }\end{array}$ & & & & & 1 & 1 \\
\hline & $\begin{array}{l}\text { Comprensión de } \\
\text { lectura }\end{array}$ & & & & & 1 & 1 \\
\hline & Expresión oral & & & & & 1 & 1 \\
\hline & Expresión escrita & & & & & 1 & 1 \\
\hline \multirow{3}{*}{$\begin{array}{l}\text { Historia, } \\
\text { Geografía } \\
\text { y Ciencias } \\
\text { Sociales }\end{array}$} & Historia & 1 & 1 & 1 & 1 & 2 & 3 \\
\hline & Geografía & 2 & 2 & 2 & 2 & 1 & 1 \\
\hline & $\begin{array}{l}\text { Formación } \\
\text { ciudadana }\end{array}$ & 2 & 2 & 2 & 2 & 2 & 2 \\
\hline \multirow[t]{3}{*}{$\begin{array}{l}\text { Ciencias } \\
\text { Naturales }\end{array}$} & $\begin{array}{l}\text { Ciencias de la } \\
\text { Vida }\end{array}$ & 2 & 2 & 2 & 2 & 1 & 2 \\
\hline & $\begin{array}{l}\text { Ciencias Físicas y } \\
\text { Químicas }\end{array}$ & 1 & 1 & 1 & 2 & 1 & 2 \\
\hline & $\begin{array}{l}\text { Ciencias de } \\
\text { la Tierra y el } \\
\text { Universo }\end{array}$ & 1 & 1 & 1 & 1 & 1 & 1 \\
\hline
\end{tabular}




\begin{tabular}{|c|c|c|c|c|c|c|c|}
\hline \multirow[t]{2}{*}{ Artes Visuales } & $\begin{array}{l}\text { Expresar y crear } \\
\text { visualmente }\end{array}$ & 1 & 1 & 1 & 1 & 1 & 1 \\
\hline & $\begin{array}{l}\text { Apreciar y } \\
\text { responder frente } \\
\text { al arte }\end{array}$ & 0 & 0 & 0 & 0 & 0 & 0 \\
\hline \multirow[t]{3}{*}{$\begin{array}{l}\text { Educación } \\
\text { Física y Salud }\end{array}$} & $\begin{array}{l}\text { Habilidades } \\
\text { motrices }\end{array}$ & 1 & 1 & 1 & 1 & 1 & 1 \\
\hline & $\begin{array}{l}\text { Vida activa y } \\
\text { saludable }\end{array}$ & 1 & 1 & 1 & 1 & 1 & 1 \\
\hline & $\begin{array}{l}\text { Seguridad, juego } \\
\text { limpio y liderazgo }\end{array}$ & 1 & 1 & 1 & 1 & 1 & 1 \\
\hline \multirow[t]{4}{*}{ Música } & $\begin{array}{l}\text { Escuchar y } \\
\text { apreciar }\end{array}$ & 1 & 1 & 1 & 1 & 1 & 1 \\
\hline & Interpretar y crear & 0 & 0 & 0 & 0 & 0 & 0 \\
\hline & $\begin{array}{l}\text { Reflexionar y } \\
\text { contextualizar }\end{array}$ & 0 & 0 & 0 & 0 & 0 & 0 \\
\hline & $\begin{array}{l}\text { Lengua, tradición } \\
\text { oral, iconografía, } \\
\text { prácticas de } \\
\text { lectura y escritura } \\
\text { de los pueblos } \\
\text { originarios. }\end{array}$ & 1 & 1 & & & & \\
\hline \multirow[t]{3}{*}{$\begin{array}{l}\text { Lengua y } \\
\text { cultura de } \\
\text { los pueblos } \\
\text { originarios } \\
\text { ancestrales }\end{array}$} & $\begin{array}{l}\text { Territorio, } \\
\text { territorialidad, } \\
\text { identidad } \\
\text { y memoria } \\
\text { histórica de } \\
\text { los pueblos } \\
\text { originarios } \\
\end{array}$ & 1 & 1 & & & & \\
\hline & $\begin{array}{l}\text { Cosmovisión } \\
\text { de los pueblos } \\
\text { originarios }\end{array}$ & 1 & 1 & & & & \\
\hline & $\begin{array}{l}\text { Patrimonio, } \\
\text { tecnologías, } \\
\text { técnicas, ciencias } \\
\text { y artes ancestrales } \\
\text { de los pueblos } \\
\text { originarios }\end{array}$ & 1 & 1 & & & & \\
\hline \multirow[t]{2}{*}{$\begin{array}{l}\text { Lengua } \\
\text { indígena }\end{array}$} & Oralidad & & & 1 & 1 & 1 & 1 \\
\hline & $\begin{array}{l}\text { Comunicación } \\
\text { escrita }\end{array}$ & & & 1 & 1 & 1 & 1 \\
\hline
\end{tabular}

Fuente: Elaborada a partir de MINEDUC (s/f)

Según la tabla, no se observan mayores diferencias entre las cantidades de OA por eje, existiendo cierto equilibrio en la selección de aprendizajes esenciales. Una excepción ocurre en Lenguaje y Comunicación de $5^{\circ}$ y $6^{\circ}$ básico, resultando perjudicado el eje Comunicación 
Oral. La diferencia entre la cantidad de OA priorizados de este eje respecto de Lectura y Escritura no existe en las BC vigentes.

Además se evidencia que los OA de los ejes de Lectura y Escritura de Lenguaje y Comunicación, Números y Operaciones de Matemática, e Historia de Historia, Geografía y Ciencia Sociales aumentan en cantidad a medida que avanzan de nivel, suponiendo una mayor profundización en estos aprendizajes. Mientras en Artes Visuales y Música existen ejes sin representación curricular (uno y dos, respectivamente), en todos los niveles.

En Matemática el eje Datos y Probabilidades está ausente en $1^{\circ} \mathrm{y}$ $2^{\circ}$ básico. Es destacable notar que este eje es el mismo que presentó la menor cobertura curricular y tiempo dedicado en Matemática, y en los mismos niveles, en el Estudio de implementación curricular en Enseñanza Básica. Similarmente sucede con los ejes no considerados en Artes Visuales. Esto ratificaría, mediante la priorización curricular, una menor estima sobre estos aprendizajes, pudiendo evaluarse positivamente si es que lo que se busca es disminuir la densidad del curriculum escolar con base en la evidencia que reportan los estudios, además de la opinión técnica. No obstante, es todavía aventurado asegurar lo antes dicho, pues MINEDUC solo describe el proceso, mas no los insumos que fundamentan la priorización de cada OA y eje curricular.

\section{A modo de cierre: La necesidad de un curriculum oficial reducido}

La priorización curricular elaborada por MINEDUC confirma la existencia de un curriculum escolar amplio, posible de ser condensado y jerarquizado en metas educativas más esenciales que otras, según necesidades de corto y largo plazo (Baeza, 2020). En lo factual se observa al currículo chileno como una construcción sujeta al contexto temporal y territorial de aplicación, alejándose de una identidad curricular meramente técnica, en la que los OA se mantienen intactos a pesar de las circunstancias. Sin embargo, este curriculum priorizado es solo una propuesta, y las $\mathrm{BC}$ permanecen legalmente vigentes junto con su robustez ya mostrada.

El exceso de saberes a enseñar en las actuales BC no responde exclusivamente al interés estatal por el control educativo. Además, 
se busca responder convincentemente "a la explosión de intereses, expectativas y necesidades que se ciernen, muchas veces caóticamente, sobre el mismo" (Amadio et al., 2015, p. 5), bajo una ideología democratizadora del curriculum (Magendzo, 2008; Gazmuri, 2017), incorporando a la mayor cantidad de visiones y perspectivas de sociedad presentes en Chile. Tal diversidad ha terminado abultando la cantidad de contenidos a enseñar, sin controlar la viabilidad de su efectiva enseñanza en el aula. De esta forma, la necesidad por configurar consensos sociales en el curriculum nacional en un civismo comprendido como paz social - a través de la elusión de conflictos, al excluir hipotéticamente un contenido-, sería el principal factor que explica los problemas derivados de la sobrecarga curricular, a saber, baja cobertura y profundidad en el aprendizaje, descontextualización de la enseñanza y barreras a la autonomía curricular de las comunidades escolares.

Del mismo modo, la saturación de metas educativas en el currículo oficial impacta en la calidad educativa. Un curriculum amplio genera frustración en el profesorado y alumnado, al verse enfrentados a "cantidades considerables de contenidos a los que no siempre consiguen atribuir sentido" (Coll, 2006, p. 5), reforzando una perspectiva transmisiva de la enseñanza y complejizando, además, la atención a la diversidad.

Por otro lado, la carga curricular es clave para favorecer o entorpecer la autonomía curricular. Si las escuelas requieren dar más contexto y sentido a lo que enseñan, un curriculum amplio, sometido a un accountability escolar mediante el SIMCE, con efectos en la oferta y demanda de matrículas, deja pocos espacios para la autonomía. De este modo, por su amplitud, la propia estructura curricular nacional termina, primero, levantando un hiato entre diseño curricular centralizado y desarrollo en la escuela, y, segundo, otorgando un gran peso en el curriculum nacional (Cárdenas et al., 2021).

Habrá que agregar que la tendencia de los currículos en las últimas décadas en el mundo se ha dirigido a la formulación de parámetros amplios, generales y abiertos, con una cantidad acotada de metas educativas, muy abiertas a la gestión y complemento de las escuelas (PREAL, 2009; UNICEF, 2013). 
En suma, en el caso chileno, un nuevo curriculum escolar, con una menor densidad curricular y mayor espacio de flexibilidad, repercutiría positivamente en:

- Adecuada cobertura curricular que posibilite un abordaje profundo de sus OA con el alumnado.

- Disminución de la saturación laboral del profesorado, asociada al cumplimiento de metas educativas.

- Mayor autonomía curricular de la escuela, comunidad escolar incluida, asegurando su función complementaria al curriculum oficial, cumpliendo lo planteado por la LGE.

- Protagonismo de las comunidades escolares al definir los elementos distintivos consagrados en sus proyectos educativos.

- Contextualización y pertinencia del curriculum, al descentralizar su gestión.

La actual contingencia pandémica ha obligado al mundo académico, escolar y docente chileno a replantear seriamente la arquitectura del currículo oficial, a una década del inicio de su implementación. Esto se amplifica en que el futuro educativo, a corto y mediano plazo, se vislumbra bajo un modalidad híbrida presencial-virtual, requiriéndose de un ajuste curricular que permita centrarse en conceptos claves y contenido esencial (Opertti, 2021). Esto debe ser visto como una oportunidad, como recomienda la OCDE: "Tal vez lo más importante sea que sepamos aprovechar el momento para que los currículos y los entornos de aprendizaje se ajusten más a las necesidades del siglo XXI" (Reimers \& Schleicher, 2020, p. 10).

No obstante, la inquietud mayor está en la disposición del poder central para estas discusiones y para tomar las decisiones que permitan a las comunidades escolares mayor autonomía, flexibilidad y contextualización de las definiciones curriculares.

\section{Referencias}

Amadio, M., Opertti, R. y Tedesco, J.C. (2015). El currículo en los debates y en las reformas educativas al horizonte 2030: Para una agenda curricular del siglo XXI. IBE Working Papers on $\mathrm{Cu}-$ rriculum Issues No 15. UNESCO-IBE. Recuperado de https:// bit.ly/2V9geh5 
Arias, K. y Quintriqueo, S. (2020). Educación superior en contexto mapuche: El caso de La Araucanía, Chile. Educare, 24(2), 1-19. DOI: http://doi.org/10.15359/ree.24-2.1

Arratia, A., Vargas, E. y Latorre, M. (2018). Alcances de la definición del curriculum nacional: desafíos de una política de desarrollo curricular. En Ministerio de Educación. República de Chile / Organización de las Naciones Unidas para la Educación, la Ciencia y la Cultura (UNESCO-Santiago) Políticas para el Desarrollo del Curriculum: Reflexiones y Propuestas (pp. 85116). Recuperado de https://cutt.ly/fkv9SGP

Baeza, A. (2020, 30 de mayo). La "priorización curricular" como el primer paso para reformar el curriculum escolar. El Mostrador. Recuperado de https://cutt.ly/ekv9JeI

Bernstein, B. (1985). Clasificación y Enmarcación del Conocimiento Educativo. Revista Colombiana de Educación, 15, 1-22. DOI: https://doi.org/10.17227/01203916.5118

Bolívar, A. (2008). Didáctica y curriculum: De la modernidad a la postmodernidad. Aljibe.

Cárdenas, C., Guerrero, S. y Johnson, D. (2021). Construir curriculum desde abajo: avanzando en la documentación de una propuesta curricular en el contexto del COVID-19. Educación, 30(58), 34-58. DOI: https://doi.org/10.18800/educacion.202101.002

Castillo, H. y Martínez, M. V. (2017). ¿En qué usan los establecimientos sus horas de libre disposición? Análisis de la Encuesta Horas de Libre Disposición 2017 en establecimientos con Jornada Escolar Completa. Documento de trabajo No 11. Centro de Estudios Mineduc. Recuperado de https://bit.ly/3ktXVLs

Castillo, V. y Salgado, V. (2018). Un desarrollo curricular inclusivo: justicia social y diferencias frente a la idea del mínimo cultural común. En Ministerio de Educación. República de Chile / Organización de las Naciones Unidas para la Educación, la Ciencia y la Cultura (UNESCO-Santiago), Políticas para el Desarrollo del Curriculum: Reflexiones y Propuestas (pp. 221246).

Centro de Estudios Mineduc (2013). Implementación del curriculum de Educación Media en Chile. En Serie Evidencias, 2(21), 1-8. Recuperado de https://bit.ly/3aZymyK 
CEPAL-UNESCO. (2020). Informe COVID-19. La educación en tiempos de la pandemia de COVID-19. Recuperado de https://bit. ly/2Vq9YRR

Colegio de Profesoras y Profesores de Chile. (2020). Orientaciones para el trabajo pedagógico a partir del Plan Educativo de Emergencia para enfrentar la Crisis Sanitaria del Colegio de Profesoras y Profesores de Chile. Recuperado de https://bit. ly/2O86saQ

Coll, E. (2006). Lo básico en la educación básica. Reflexiones en torno a la revisión y actualización del currículo de la educación básica. Revista Electrónica de Investigación Educativa, 8(1), 1-17. https://bit.ly/3dOXzOt

Consejo Nacional de Educación de Chile (CNED). (2020, 13 de mayo). Acuerdo $\mathrm{N}^{\circ}$ 080/2020. Recuperado de https://bit.ly/2P9l22m

Contreras, G. (2014). Caracterización del curriculum evaluado en matemática en sexto año básico. Un estudio descriptivo en Valparaíso, Chile. RELIEVE, 20(2), 1-27. DOI: https://doi. org/10.7203/relieve.20.2.4295

Cox, C. (2018). Curriculum: categorías de análisis, tendencias, gobernanza. En Ministerio de Educación. República de Chile / Organización de las Naciones Unidas para la Educación, la Ciencia y la Cultura (UNESCO-Santiago). Políticas para el Desarrollo del Curriculum: Reflexiones y Propuestas (pp. 119$154)$.

CNN Chile. (2020, 05 de mayo). Cronología del COVID-19: Todos los hitos claves en Chile y el mundo desde el 31/12/19. Recuperado de https://bit.ly/2NOwOii

Díaz Barriga, A. y García, J. M. (2014). Introducción. En A. Díaz Barriga y J. M. García (Eds.), Desarrollo del curriculum en América Latina. Experiencia de diez países. El curriculum en América Latina. Caleidoscopio de una región (pp. 09-14). Miño y Dávila.

Dussel, I. (2020). La clase en pantuflas. En I. Dussel, P. Ferrante y D. Pulfer (compiladores), Pensar la educación en tiempos de pandemia: entre la emergencia, el compromiso y la espera. UNIPE. Recuperado de https://bit.ly/3if8d2L

Espinoza, O. (2016). Cambios al curriculum escolar 1990-2014: institucionalidad y desafíos. CEPPE Policy Brief, 7. Recuperado de https://bit.ly/2ZUQCTA 
Gazmuri, R. (2017). Ideologías curriculares en el debate y negociación del curriculum chileno de Historia, Geografía y Ciencias Sociales. Estudios Pedagógicos, XLIII(1), 157-169. DOI: http:// dx.doi.org/10.4067/S0718-07052017000100010

Grundy, S. (1998). Producto o praxis del curriculum. Morata.

Haas, V., Molina, E., Bravo, J. y Manghi, D. (2016). Contraste entre el Curriculum Nacional, Texto Escolar y las decisiones del profesor en el aula: Análisis del discurso desde la perspectiva multimodal en clases de Historia y Geografía. Profesorado: Revista de Curriculum y Formación del Profesorado, 20(3), 367-397. Recuperado de https://bit.ly/3pU4WGI

Inostroza, C., y Lohaus, M. (2019). Inclusión y diversidad: nuevos desafíos para la política curricular chilena. reflexiones desde la teoría curricular y la justicia social. Revista Internacional de Educación para la Justicia Social, 8(1), 151-162. DOI: https:// doi.org/10.15366/riejs2019.8.1.009

Ley $\mathrm{N}^{\circ}$ 20.370. Establece la Ley General de Educación. 12 de septiembre de 2009. Diario Oficial de la República de Chile, № 39.461. Recuperado de https://bit.ly/3sybXyx

Magendzo, A. (2008). Dilemas del curriculum y la pedagogía. Analizando la Reforma Curricular desde una perspectiva crítica. LOM.

Marrero, J. (2010). El curriculum que es interpretado. ¿Qué enseñan los centros y los profesores y profesoras? En J. Gimeno Sacristán (Comp.), Saberes e incertidumbre sobre el curriculum (pp. 221-245). Morata.

Mayo Clinic (s/f). Enfermedad del coronavirus 2019 (COVID-19). Recuperado el 9 de enero de $2021 \mathrm{de} \mathrm{https://mayocl.in/3q1ROza}$

Ministerio de Educación, Mineduc. (2015). Estudio de implementación curricular en Enseñanza Básica: Reportes individuales. Recuperado de https://bit.ly/2NC0EXx

Ministerio de Educación, Mineduc. (2016). Recomendaciones para una Política Nacional de Desarrollo Curricular. Informe Mesa de Desarrollo Curricular. Recuperado de https://bit. ly/3pYnFkw

Ministerio de Educación, Mineduc. (2018a). Bases Curriculares Primero a Sexto Básico. Recuperado de https://bit.ly/3sy4rUy

Ministerio de Educación, Mineduc. (2018b). Estudio de Exploración y Análisis de los Procesos de Implementación Curricular en el 
Sistema Educacional Chileno. Informe Final. Recuperado de https://bit.ly/3bISlkt

Ministerio de Educación, Mineduc. (2020a, 25 de marzo). Se extiende período de suspensión de clases. Recuperado de https://bit. $\mathrm{ly} / 3 \mathrm{bISc} 0 \mathrm{p}$

Ministerio de Educación, Mineduc. (2020b). Fundamentación Priorización Curricular Covid-19. Recuperado de https://bit. ly/3b25LZX

Ministerio de Educación, Mineduc. (2020c). Orientaciones para la Implementación de la Priorización Curricular en Forma Remota y Presencial. Recuperado de https://bit.ly/3b1Nwn6

Ministerio de Educación, Mineduc. (s/f). Bases Curriculares. Recuperado el 02 de enero de 2021. https://bit.ly/37PPJjx

Oliva, M. A. (2017). Arquitectura de la Política Educativa Chilena (1990-2014): El curriculum, lugar de la metáfora. Revista Brasileira de Educação, 22(69), 405-428. DOI: http://dx.doi. org/10.1590/s1413-24782017226921

Opertti, R. (2021). Diez pistas para repensar el curriculum. Reflexiones en Progreso No 42. IBE-UNESCO. Recuperado de https://bit. ly/3rRhDEP

Organización de las Naciones Unidas para la Educación, la Ciencia y la Cultura (UNESCO-OIE). (2013). Herramientas de Formación para el Desarrollo Curricular: Una Caja de Recursos. Recuperado de https://bit.ly/3b01HcE

Ossandón, L. y Pinto, R. (2018). El curriculum nacional y la descentralización: políticas, institucionalidad y saberes. En Ministerio de Educación. República de Chile / Organización de las Naciones Unidas para la Educación, la Ciencia y la Cultura (UNESCO-Santiago), Políticas para el Desarrollo del Curriculum: Reflexiones y Propuestas (pp. 155-186).

Pascual, E. (1998). Racionalidades en la Producción Curricular y el Proyecto Curricular. Pensamiento Educativo, (23), 13-72. Recuperado de https://bit.ly/3aZPmFj

Programa de Promoción de la Reforma Educativa en América Latina y el Caribe (PREAL). (2009). Tendencias en la evaluación del logro escolar: la experiencia de cinco países con alto rendimiento educativo. Serie Mejores Prácticas / Formas y Reformas de la Educación, 11(31), 1-4. Recuperado de https:// cutt.ly/tkv9LQ3 
Reimers, F. y Schleicher, A. (2020). Aprendiendo durante la pandemia. De la disrupción a la innovación. Versión preliminar. OCDE. Recuperado de https://bit.ly/3loJs65

Rodríguez, D. (2018). El curriculum y la libertad de enseñanza: aproximaciones desde una perspectiva liberal. En Ministerio de Educación. República de Chile / Organización de las Naciones Unidas para la Educación, la Ciencia y la Cultura (UNESCO-Santiago), Políticas para el Desarrollo del Curriculum: Reflexiones y Propuestas (pp. 187-217).

Rodríguez, C., Castillo, V. y Saavedra, R. (2015). Expectativa, Cobertura y Dominio Curricular: Percepciones del profesorado en la enseñanza de la Matemática. Revista Paradigma, XXXVI(2), 177-201. Recuperado de https://bit.ly/3dOWbeJ

Tadeu Da Silva, T. (1999). Documentos de identidad. Una introducción a las teorías del currículo. Auténtica. 